"Mircea cel Batran" Naval Academy Scientific Bulletin, Volume XIX - 2016 - Issue 2

The journal is indexed in: PROQUEST / DOAJ / Crossref / EBSCOhost / INDEX COPERNICUS / DRJI / OAJI /

JOURNAL INDEX / I2OR / SCIENCE LIBRARY INDEX / Google Scholar / Academic Keys/ ROAD Open Access I

Academic Resources / Scientific Indexing Services / SCIPIO / JIFACTOR

\title{
FEATURES‘ANALYSIS OF SMALL AND VERY SMALL SCALE WINGS EXPERIMENTED BYAERODYNAMICS TUNNELAND CONFRUNTED WITH THE TWO SCALES SIMILARITY THEORY
}

\author{
Beazit ALI ${ }^{1}$ \\ Anastase PRUIU ${ }^{2}$ \\ Adriana SPORIS ${ }^{3}$ \\ Gheorghe ICHIMOAEI ${ }^{4}$ \\ Levent ALI ${ }^{5}$ \\ ${ }^{1}$ Professor Ph.D.Eng.,Marine Engineering and Naval Weapons Department, "Mircea cel Batan" Naval \\ Academy, Constanţa, Romania \\ ${ }^{2}$ Professor Ph.D.Eng., Marine Engineering and Naval Weapons Department, "Mircea cel Batran" Naval \\ Academy, Constanţa, Romania \\ ${ }^{3}$ Senior Lecturer Ph.D.Eng., Marine Engineering and Naval Weapons Department, "Mircea cel Batran" Naval \\ Academy, Constanţa, Romania \\ ${ }^{4}$ Lecturer PhD., "Mircea cel Batran" Naval Academy \\ ${ }^{5}$ Ph.D.attendee Eng., Bureau Veritas Romania Controle International, Romania
}

Abstract: It compares the NACA 6412 profile and the NACA 0015 profile specific features experimentally with using the two scales similarity theory. The results confirm the fact the two scales similarity theory represents a simple and method establish the hydrodynamic special features of profiles.

Keywords: similarity theory, span, model wing, distort ratio, elongation, aerodynamics coefficient

\section{Introduction}

For raising the features of small and very smallscale wings, experimental tests have been conducted on the NACA 0015 and NACA 6412 profile in the aerodynamics tunnel.

Experimental tests have been carried out at consistent flow within the measurement section $1600 \times 1200 \mathrm{~mm}$ of the naval aerodynamics tunnel for a range of angles of attack $\alpha$ comprised between $0^{\circ}$ and $32^{\circ}$ for NACA 0015 and between $10^{\circ}$ and $15^{\circ}$ for NACA 6412 (elongation $\lambda \leq 6$ ).

Determining the aerodynamic torque, overall qualitative type forces and moments have been achieved by using a strain gauge balance with six components of Kempf Remmers type. Experimental data storage for each angle has been made on the computer. By means of a software package such data has been processed thus obtaining the variation curves of the aerodynamic coefficients $c_{y}$ and $c_{x}$. The results are shown in tables 2 and 3 for NACA 0015 profile and in tables 7 and 8 for NACA 6412 profile.
Analysis by comparison of NACA 0015 profile andNACA 6412 profile features, experimentally obtained by aerodynamics tunnel with their features achieved through the two sales similarity theory

$R_{y}=F_{N} \cos \alpha-F_{T} \sin \alpha$-carrying capacity(1)

$R_{N}=F_{N} \sin \alpha-F_{T} \cos \alpha$ - resistance to progress

(2)

$C_{y}=\frac{R_{y}}{\frac{\rho v^{2}}{2} \cdot S}-$ bearing capacity coefficient

(3) $c_{x}=\frac{R_{x}}{\frac{\rho v^{2}}{2} \cdot S}-$ resistance to

progresscoefficient(4)

$S=c \cdot l$-wing surface $(5)$

The geometric parameters of NACA 0015 profile are provided in Table 1.

DOI: 10.21279/1454-864X-16-I2-021

(c) 2015. This work is licensed under the Creative Commons Attribution-Noncommercial-Share Alike 4.0 License. 
"Mircea cel Batran" Naval Academy Scientific Bulletin, Volume XIX - 2016 - Issue 2

The journal is indexed in: PROQUEST / DOAJ / Crossref / EBSCOhost / INDEX COPERNICUS / DRJI / OAJI / JOURNAL INDEX / I2OR / SCIENCE LIBRARY INDEX / Google Scholar / Academic Keys/ ROAD Open Access I Academic Resources / Scientific Indexing Services / SCIPIO / JIFACTOR

Table 1

\begin{tabular}{|l|l|}
\hline$\%$ c & $\begin{array}{l}\text { Thickness of } \\
\text { sect \% I }\end{array}$ \\
\hline 0 & 0 \\
\hline 5 & 8,8 \\
\hline 10 & 11,7 \\
\hline 20 & 14,3 \\
\hline 30 & 15 \\
\hline 40 & 14,5 \\
\hline 50 & 13,2 \\
\hline 60 & 11,4 \\
\hline 70 & 9,1 \\
\hline 80 & 6,5 \\
\hline 90 & 3,62 \\
\hline 100 & 0 \\
\hline
\end{tabular}

and aerodynamic parameters experimented by aerodynamics tunnel are provided in tables (2) and (3).

$c_{y}=f(\alpha, \lambda)$ Table 2

\begin{tabular}{|l|l|l|l|l|l|l|l|l|}
\hline$\lambda$ & & & & & & & & \\
$\alpha^{0}$ & 0,25 & 0,50 & 0,75 & 1,0 & 1,5 & 2,0 & 3,0 & 5,0 \\
\hline 0 & 0 & 0 & 0 & 0 & 0 & 0 & 0 & 0 \\
\hline 4 & 0,04 & 0,07 & 0,09 & 0,10 & 0,14 & 0,16 & 0,22 & 0,25 \\
\hline 8 & 0,1 & 0,15 & 0,19 & 0,23 & 0,29 & 0,35 & 0,45 & 0,50 \\
\hline 12 & 0,15 & 0,26 & 0,31 & 0,36 & 0,44 & 0,54 & 0,69 & 0,75 \\
\hline 16 & 0,22 & 0,36 & 0,43 & 0,50 & 0,61 & 0,73 & 0,88 & 0,95 \\
\hline 20 & 0,32 & 0,48 & 0,56 & 0,64 & 0,80 & 0,90 & 0,89 & 0,70 \\
\hline 24 & 0,41 & 0,60 & 0,72 & 0,80 & 0,93 & 0,53 & 0,56 & 0,72 \\
\hline 28 & 0,51 & 0,75 & 0,86 & 0,94 & 0,47 & 0,49 & 0,56 & 0,73 \\
\hline 30 & 0,60 & 0,88 & 1,00 & 1,05 & 0,49 & 0,50 & 0,56 & 0,74 \\
\hline
\end{tabular}

$c_{x}=f(\alpha, \lambda)$ Table 3

\begin{tabular}{|l|l|l|l|l|l|l|l|l|}
\hline $\begin{array}{l}\lambda \\
\alpha^{0}\end{array}$ & 0,25 & 0,50 & 0,75 & 1,0 & 1,5 & 2,0 & 3,0 & 5,0 \\
\hline 0 & 0,03 & 0,01 & 0,02 & 0,01 & 0,02 & 0,01 & 0,01 & 0,01 \\
\hline 4 & 0,05 & 0,02 & 0,02 & 0,02 & 0,02 & 0,02 & 0,01 & 0,01 \\
\hline 8 & 0,05 & 0,03 & 0,03 & 0,03 & 0,04 & 0,03 & 0,04 & 0,03 \\
\hline 12 & 0,07 & 0,05 & 0,06 & 0,06 & 0,06 & 0,06 & 0,07 & 0,07 \\
\hline 16 & 0,10 & 0,09 & 0,10 & 0,10 & 0,11 & 0,11 & 0,11 & 0,19 \\
\hline 20 & 0,12 & 0,14 & 0,14 & 0,15 & 0,16 & 0,16 & 0,27 & 0,25 \\
\hline 24 & 0,19 & 0,21 & 0,23 & 0,22 & 0,23 & 0,22 & 0,31 & 0,28 \\
\hline 28 & 0,26 & 0,30 & 0,30 & 0,30 & 0,28 & 0,35 & 0,36 & 0,37 \\
\hline 30 & 0,34 & 0,37 & 0,42 & 0,38 & 0,47 & 0,40 & 0,39 & 0,40 \\
\hline
\end{tabular}

The variation of the $c_{y}$ aerodynamic coefficient depending on the angle of incidence $\alpha$ of NACA0015 profile is appropriate for the elongations that it has been experienced.
Applying the two scales similarity theory of NACA0015 profile with $\lambda=5$ obtained for elongations $\lambda=$ 0,$25 ; 0,50 ; 0,75 ; 1,0 ; 1,5 ; 2,0$; and 3,0 the following

\begin{tabular}{|c|l|l|l|l|l|l|l|}
\hline $\begin{array}{c}\lambda \\
\alpha^{0}\end{array}$ & 2 & 3 & 6 & $\begin{array}{r}\lambda \\
\alpha^{0}\end{array}$ & 2 & 3 & 6 \\
\hline- & $-0,35$ & $-0,38$ & $-0,34$ & - & 0,13 & $-0,15$ & 0,17 \\
10 & & & & 10 & & & \\
\hline-8 & $-0,25$ & $-0,34$ & $-0,33$ & -8 & 0,10 & 0,12 & 0,14 \\
\hline-6 & $-0,01$ & $-0,22$ & $-0,31$ & -6 & 0,08 & 0,09 & 0,12 \\
\hline-4 & $-0,05$ & $-0,01$ & $-0,20$ & -4 & 0,08 & 0,08 & 0,09 \\
\hline-2 & 0,26 & 0,21 & $-0,06$ & -2 & 0,06 & 0,07 & 0,08 \\
\hline 0 & 0,45 & 0,52 & 0,40 & 0 & 0,07 & 0,08 & 0,09 \\
\hline 3 & 0,76 & 0,92 & 1,10 & 3 & 0,12 & 0,13 & 0,12 \\
\hline 6 & 1,01 & 1,23 & 1,55 & 6 & 0,17 & 0,20 & 0,17 \\
\hline 9 & 1,26 & 1,50 & 1,90 & 9 & 0,25 & 0,29 & 0,28 \\
\hline 12 & 1,51 & 1,78 & 2,14 & 12 & 0,36 & 0,39 & 0,36 \\
\hline 15 & 1,74 & 1,96 & 1,61 & 15 & 0,49 & 0,53 & 0,44 \\
\hline
\end{tabular}

values of the bearing capacity $c_{y}$ coefficient and the resistance to progress $C_{x}$ the coefficient are shown in Tables 4 and 5.

$c_{y}=f(\alpha, \lambda)$ Table 4

\begin{tabular}{|l|l|l|l|l|l|l|l|l|}
\hline $\begin{array}{r}\lambda \\
\alpha^{0}\end{array}$ & 0,25 & 0,50 & 0,75 & 1,0 & 1,5 & 2,0 & 3,0 & 5,0 \\
\hline 0 & 0 & 0 & 0 & 0 & 0 & 0 & 0 & 0 \\
\hline 4 & 0,01 & 0,02 & 0,04 & 0,05 & 0,07 & 0,10 & 0,15 & 0,25 \\
\hline 8 & 0,02 & 0,05 & 0,07 & 0,10 & 0,15 & 0,20 & 0,30 & 0,50 \\
\hline 12 & 0,04 & 0,07 & 0,11 & 0,15 & 0,22 & 0,30 & 0,45 & 0,75 \\
\hline 16 & 0,05 & 0,09 & 0,14 & 0,19 & 0,28 & 0,38 & 0,57 & 0,95 \\
\hline 20 & 0,03 & 0,07 & 0,11 & 0,14 & 0,21 & 0,28 & 0,42 & 0,70 \\
\hline 24 & 0,04 & 0,07 & 0,11 & 0,14 & 0,22 & 0,29 & 0,43 & 0,72 \\
\hline 28 & 0,04 & 0,07 & 0,11 & 0,15 & 0,22 & 0,29 & 0,44 & 0,73 \\
\hline 30 & 0,05 & 0,07 & 0,11 & 0,15 & 0,22 & 0,30 & 0,44 & 0,74 \\
\hline
\end{tabular}

$c_{x}=f(\alpha, \lambda)$ Table 5

\begin{tabular}{|l|l|l|l|l|l|l|l|l|}
\hline $\begin{array}{l}\lambda \\
\alpha^{0}\end{array}$ & 0,25 & 0,50 & 0,75 & 1,0 & 1,5 & 2,0 & 3,0 & 5,0 \\
\hline 0 & 0,00 & 0,00 & 0,00 & 0,00 & 0,00 & 0,00 & 0,00 & 0,01 \\
\hline 4 & 0,00 & 0,00 & 0,00 & 0,00 & 0,00 & 0,01 & 0,01 & 0,01 \\
\hline 8 & 0,00 & 0,00 & 0,00 & 0,00 & 0,01 & 0,01 & 0,02 & 0,03 \\
\hline 12 & 0,00 & 0,01 & 0,01 & 0,01 & 0,02 & 0,03 & 0,04 & 0,07 \\
\hline 16 & 0,01 & 0,02 & 0,03 & 0,04 & 0,06 & 0,08 & 0,12 & 0,19 \\
\hline 20 & 0,01 & 0,03 & 0,04 & 0,05 & 0,07 & 0,10 & 0,15 & 0,25 \\
\hline 24 & 0,01 & 0,03 & 0,04 & 0,06 & 0,08 & 0,11 & 0,17 & 0,28 \\
\hline 28 & 0,02 & 0,04 & 0,06 & 0,07 & 0,11 & 0,15 & 0,22 & 0,37 \\
\hline 30 & 0,02 & 0,04 & 0,06 & 0,08 & 0,12 & 0,16 & 0,24 & 0,40 \\
\hline
\end{tabular}

For NACA 6412 profile, the geometric parameters are provided in Table 6. 
"Mircea cel Batran" Naval Academy Scientific Bulletin, Volume XIX - 2016 - Issue 2

The journal is indexed in: PROQUEST / DOAJ / Crossref / EBSCOhost / INDEX COPERNICUS / DRJI / OAJI /

JOURNAL INDEX / I2OR / SCIENCE LIBRARY INDEX / Google Scholar / Academic Keys/ ROAD Open Access I Academic Resources / Scientific Indexing Services / SCIPIO / JIFACTOR

Table 6

\begin{tabular}{|l|l|l|}
\hline \% c & $\begin{array}{l}\text { extrados } \\
\% ~ c\end{array}$ & $\begin{array}{l}\text { intrados } \\
\% \text { c }\end{array}$ \\
\hline 0,0 & 0,0 & 0,0 \\
\hline 5,0 & 5,36 & $-1,99$ \\
\hline 10 & 7,58 & $-1,99$ \\
\hline 20 & 10,3 & $-1,25$ \\
\hline 30 & 11,6 & $-0,38$ \\
\hline 40 & 11,8 & 0,2 \\
\hline 50 & 11,1 & 0,55 \\
\hline 60 & 9,9 & 0,8 \\
\hline 70 & 8,2 & 0,8 \\
\hline 80 & 6,0 & 0,7 \\
\hline 90 & 3,3 & 0,39 \\
\hline 100 & 0,0 & 0,0 \\
\hline
\end{tabular}

and the aerodynamic parameters experimented by aerodynamics tunnel on $\mathrm{Re}=85.000$, are provided in

Table 7.

$c_{y}=f(\lambda, \alpha, \mathrm{Re}) ; c_{x}=f(\lambda, \alpha, \mathrm{Re})$ Table 7

The polarities of NACA 6412 profile corresponding to the elongation $\lambda=2,3$ and 6 and number $\operatorname{Re}=$ 85.000 are shown in figure 3 , below.

\begin{tabular}{|l|l|l|l|l|l|l|l|}
\hline$\lambda$ & & & & $\begin{array}{l}\lambda \\
\alpha^{0}\end{array}$ & 2 & 3 & 6 \\
\hline-0 & $-0,11$ & $-0,17$ & $-0,34$ & - & 0,06 & 0,08 & 0,17 \\
& & & & 10 & & & \\
\hline-8 & $-0,11$ & $-0,17$ & $-0,33$ & -8 & 0,04 & 0,07 & 0,14 \\
\hline-6 & $-0,10$ & $-0,16$ & $-0,31$ & -6 & 0,04 & 0,06 & 0,12 \\
\hline-4 & $-0,07$ & $-0,10$ & $-0,20$ & -4 & 0,03 & 0,05 & 0,09 \\
\hline-2 & $-0,02$ & $-0,03$ & $-0,06$ & -2 & 0,06 & 0,04 & 0,08 \\
\hline 0 & 0,13 & 0,2 & 0,39 & 0 & 0,03 & 0,04 & 0,09 \\
\hline 3 & 0,36 & 0,55 & 1,10 & 3 & 0,04 & 0,06 & 0,12 \\
\hline 6 & 0,51 & 0,78 & 1,54 & 6 & 0,06 & 0,08 & 0,17 \\
\hline 9 & 0,63 & 0,95 & 1,90 & 9 & 0,09 & 0,14 & 0,28 \\
\hline 12 & 0,71 & 1,07 & 2,14 & 12 & 0,12 & 0,18 & 0,36 \\
\hline 15 & 0,54 & 0,80 & 1,61 & 15 & 0,15 & 0,22 & 0,44 \\
\hline
\end{tabular}

Applying the two scales similarity method to NAC 6412 profile too with $\lambda=6$, we obtain for elongations $\lambda=2$ and $\lambda=3$ the following values of the bearing capacity $c_{y}$ coefficient and the one of the resistance to progress $c_{x}$ coefficient in table 8 , valid for a number $\operatorname{Re} . \operatorname{Re}=85000 \cdot \frac{K_{c}^{2}}{\sqrt{K_{l}}}$

$$
\left.c_{y}=f(\lambda, \alpha, \mathrm{Re})\right) ; c_{x}=f(\lambda, \alpha, \mathrm{Re}) \text { Table } 8
$$

\section{CONCLUSIONS}

By comparing the values obtained for the two profiles with those experimentally obtained by wind tunnel, a dispersion of the experimental values is observed related to the values of those calculated by the two scales similarity method since the experiments were conducted at Reynolds numbers that differ from those obtained by applying the theory of similarity between the two scales or otherwise similarity values obtained by applying the two scales similarity method being valid for another Reynolds number which differs from that of the model. This aspect related to the Reynolds number on the model and on the prototype model, it results even from the law of this phenomenon.

\section{BIBLIOGRAPHY}

[1]."Aerodynamic tunnel tests for determining the aerodynamic features of profiles", Contract no. 621 , Galaţi 2007.

[2].NIESTOJ, W, Profiles for aeromodels, Warsaw, 1974.

[3].Vasilescu, AL. A., Analiză dimensională şi teoria similitudinii, Editura Academiei,Bucureşti, 1969.

[4].Vasilescu, AL. A., Similitudinea sistemelor elastice, Editura Academiei,Bucureşti, 1969.

[5].Carafoli, E, Constantinescu, V. N., Dinamica fluidelor incompresibile, Editura Academiei, Bucureşti, 1981.

[6].Beazit Ali, Stabilirea punţii de legătură între teoria aripii de mică anvergură şi teoria aripii de mare anvergură pe fondul teoriei similitudinii la două scări, Referatde doctorat, Universitatea "Dunărea de Jos" Galaţi, 1995.

[7].Beazit Ali, Obţinerea polarelor aripilor de mică anvergură plecând de la polarele aripilor de mare anvergură, folosind teoria similitudinii la două scări, Buletinul,,Tehmar”, Constanţa, 1996.

[8]. Beazit Ali, Traian Florea, Study on the upward small span profile based on the two scale similarity theory, The XII-th National Conference onThermotechnicswith International Participation, Naval Academy "Mircea cel Bătrân",Constanta, 2002.

DOI: 10.21279/1454-864X-16-I2-021

(C) 2015. This work is licensed under the Creative Commons Attribution-Noncommercial-Share Alike 4.0 License. 